\title{
\begin{tabular}{|l|l|} 
IV Simpósio Paranaense de Modelagem, & Artigo: \\
$\begin{array}{c}\text { Simulaçãoe Controle de Processos } \\
\text { ISSN : 1984-7521 }\end{array}$ & Páginas: 226 - 232
\end{tabular}
}

\section{Wavelet Transform as a tool in the monitoring of fermentative processes through image processing}

Jair Certório, Matheus Giovanni, Renam Luiz Acorsi, Eugênia Leandro Almeida*, José Eduardo Olivo and Cid Marcos Andrade

Department of Chemical Engineering - State University of Maringá

E-mail: eugenia-almeida@ hotmail.com

\begin{abstract}
The purpose of this work was to propose a methodology for monitoring fermentative processes through image processing. The growth of the microorganisms during the fermentation process was evaluated in this work. The images were collected in situ from time to time. At these same time intervals samples of the fermentative medium were removed, analytically measured the concentrations of microorganisms. Using the Wavelet Transform with the objective of searching for patterns that can correlate to the analytical measures of concentration of microorganisms with the images taken during the fermentation process. The results were satisfactory from the point of view of the correlations found between the data. We can conclude that the methodology is possible, but it needs improvements.
\end{abstract}

Keywords: Fermentation processes, Image processing, monitoring.

\section{Introduction}

The use of the Wavelet Transform in image processing is promising. For, with a smaller amount of values can represent the whole image. These values can be used in the assembly of the characteristics vector to represent an image $[3,4,5]$.

The theory of Wavelet Transform is applicable in different branches of science. Wavelet is a waveform with limited duration and an average value equal to zero. The Waveform Transformation provides a precise representation of a signal as a function of time and frequency simultaneously, as well as allows analyzing any type of signal, be it non- stationary or discontinuous, which proves the thesis that for its use, no matter what the characteristics of the series being analyzed. This is even a great advantage that has motivated the use of the Wavelet Transform in large scale for numerous applications and of diverse nature, since this would not be possible to be using the existing traditional techniques for analysis of time series.

The definition of Wavelet Transform considering a continuous signal is given by:

$$
F(a, b)=\int f(t) \psi_{a . b} d t
$$

\section{4 e 15 de março de 2019 Curitiba - Paraná}


In the current context, there is a growing need to produce biofuels, depending on several aspects, among them: fossil fuels are finite; their use contributes to environmental pollution and worsening global warming. The production of many liquid or gaseous biofuels is via fermentation processes. It is possible to optimize the production of these biofuels by improving the yields of fermentation processes [1].

The fermentation processes were monitored, mostly, analytically, taking samples from time to time. In order to obtain greater efficiency of these processes, studies have been developed with the objective of monitoring in real time (on- line) and on-site (in situ) concentration values that can be used to control and optimize the process, prevent infections in the middle, among other possibilities [2].

In this equation the parameters $a$ and $b$ vary continuously in $\mathrm{R}$, being the functions $\Psi a, b$ are referred to as wavelets and are defined as follows:

$$
\psi_{a, b}(t)=\frac{1}{\sqrt{a}} \psi \frac{(t-b)}{a}
$$

The Wavelet Transform for discrete signals is defined by:

$$
F_{m, n}(a, b)=a_{0}{ }^{-m / 2} \int f(t) \psi\left(a_{0}^{-m} t-n b_{0}\right) d t
$$

One form of Wavelet Transforms is the one developed by Haar (Meyer, 1993) and that we use in this work. It is described on an orthonormal base of waveforms defined on the domain $[0,1]$, that is, $\mathrm{h} 0, \mathrm{~h} 1, \ldots, \mathrm{hn}$ such that for any continuous function $\mathrm{f}(\mathrm{x})$ on the interval $[0,1]$, the series:

$$
\sum_{j=1}^{\infty}<f, h_{j}>h_{j}(x)
$$

Converges $f(x)$ evenly over $[0,1]$. Here $\langle u, v>$ represents the inner product of $\mathrm{u}$ and $\mathrm{v}$.

$$
<u, v>=\int_{n}^{1} u(x) \bar{v}(x) d x
$$

Where $\bar{v}$ is the complex conjugate of which is equal to if the function is real.

One version of Haar's construction is as follows:

$$
\begin{gathered}
h(x)\left\{\begin{array}{c}
0, x<0 \\
1,0<x<\frac{1}{2} \\
-1, \frac{1}{2}<x<1 \\
0, x>1
\end{array}\right. \\
h_{n}(x)=2^{j / 2}\left(2^{j} x-k\right)
\end{gathered}
$$

Where $n=2 j+k, k \in[0,2 j), x \in[k 2-j,(k+1) 2-1)$.

The objective of this work was to develop a methodology that allows associating images taken during the fermentation process with the microorganism concentration. The images were captured at the times of 15, 30, 45 and 60 seconds. At these same time intervals, a sample of the fermentative medium was withdrawn, to 
analyze analytically the concentration of microorganisms. For the development of this work we used experimental apparatus, as well as mathematical tools, such as the wavelet transform and computational tools, such as the Python program and Scilab $[6,7]$.

\section{Materials and methods}

The synthetic culture medium, proposed by Gutierres (1993), was prepared. Discontinuous fermentations were performed to obtain data on growth/concentration of microorganism [8]. The microorganism used in the growth tests was the yeast Saccharomyces cerevisae.

The bioreactor used BIOSTET ${ }^{\circledR} \mathrm{B}$ to carry out the fermentations. This bioreactor has agitation control unit and temperature of the fermentative medium. This bioreactor is equipped with an automatic sampler preventing contamination during sample withdrawals.

During the fermentation process samples were withdrawn at the intervals of 15, 30, 45 and 60 minutes. All measurements were performed in triplicates.

The quantification of Saccharomyces cerevisiae in the fermentative medium was done by gravimetrically. That gravimetric quantification was performed using graduated centrifuge tubes. Immediately after centrifugation, the total volume of centrifuged sample and the total volume of decanted were read. From this, the volume percentage of yeast from the medium is known. According to Borzani (2006) one can consider the specific mass of cells identical to that of the culture medium [9].The camera was added to the bioreactor vessel as shown in figure 1.

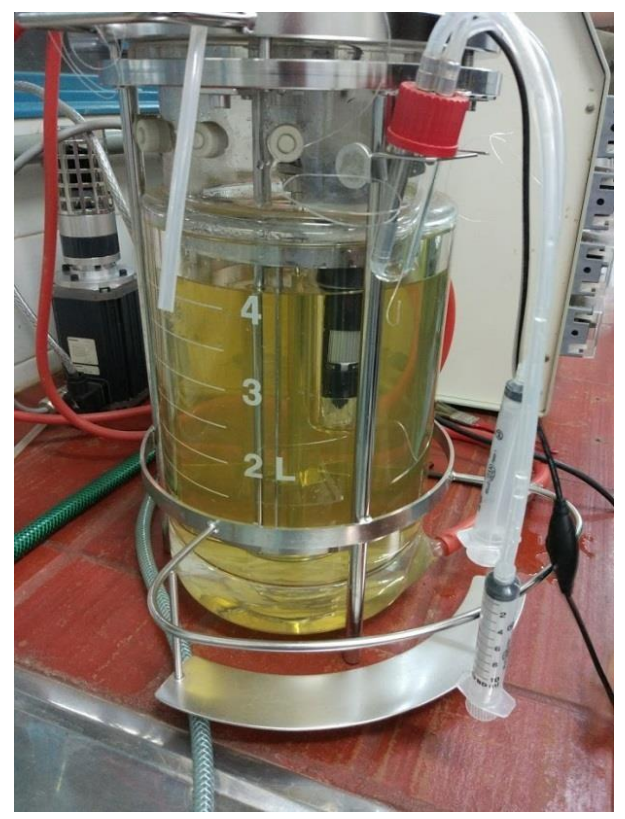

Figure 1: Bioreactor vessel containing culture medium, sampler and the camera properly conditioned.

The photos were captured in the same time interval as samples from the culture medium. 
The conversion of JPEG to CSV format was performed using a Python program. This conversion made it easy to read in the Scilab numerical program, which has the native ability to read such files. Being the image of $640 \times 480$ decomposed into three colors: red, green and blue. Matrices of 640 x 1440 were generated with each matrix of one of the stacked colors.

\section{Results and discussion}

The quantification of Saccharomyces cerevisae in the fermentative medium was done gravimetrically. With the volumes of decanted in the centrifuge tubes, the cell concentration values found in each assay were calculated. The results are shown in figure 2.

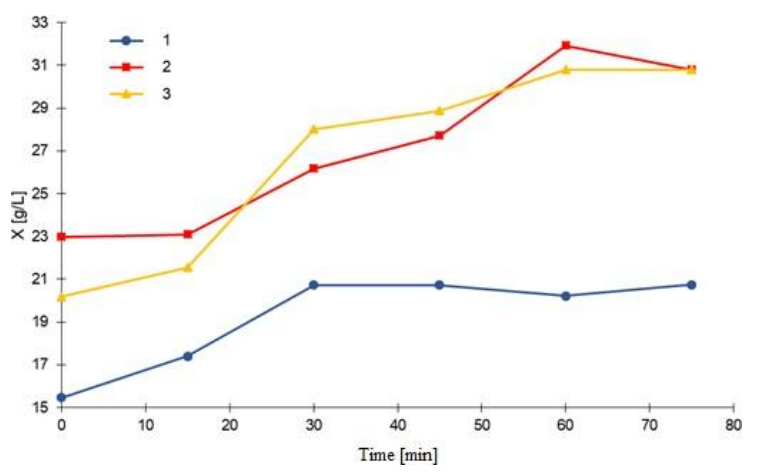

Figure 2: Cell growth of fermentation assays performed.

In the fermentative test 1 , there was a large flocculation of the yeast, causing it to form large lumps in the culture medium. Yeast flocculation is a relatively common occurrence in many situations. However, this flocculation makes sampling difficult, because with such a situation, we have fewer yeasts dissolved in the middle. Unwanted fluctuations in the value of cell concentration may also occur, as some of these lumps may be sucked up by the sampler. It is noteworthy that the fermentative test 1 also presented intense foam formation, which is another factor that hampers the sampling, in addition to the presence of yeast flakes in this foam.

Assays 2 and 3 occurred without flocculation and foaming was not as intense as that observed in test 1 . No problems were noted during the course of these two fermentation tests.

The images captured during the fermentation process to evaluate cell growth are presented in figure 3. 


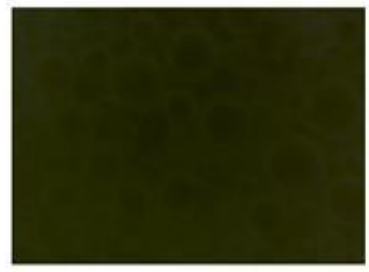

15 minutes

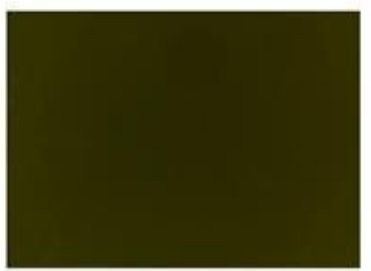

45 minutes

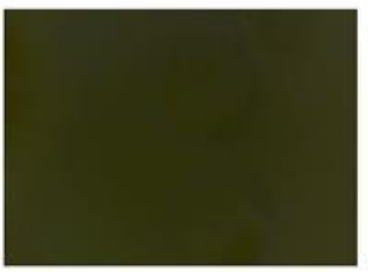

30 minutes

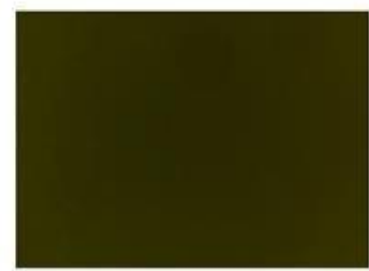

60 minutes

Figure 3: Photos taken during cell growth at 15, 30, 45 and 60 minutes.

For each image, previously converted to JPG in CSV, the walvelet transform of Haar was applied, which in the implementation of Scilab returns four matrices. The lower frequency (low pass filter), horizontal (in-line), vertical (column-by-column) and diagonal $[10,11]$.

In order to correlate with the analytical values of the resulting matrices, several functions were applied, among them: the sum of each element, largest element, least element and determinant. Being the same applied in the transform of each image, forming a time series by type of function used. Then, the various series were correlated with the measured values and it was found that the best result was the largest element of the vertical variation. The values of $0.9064971,0.8242512$ and 0.4430352 for each experiment were reached, and the comparative graph between the values measured analytically and the values obtained from the largest element of the vertical variation matrix can be seen in Figures 4, 5 and 6 respectively for experiments 1,2 and 3 .

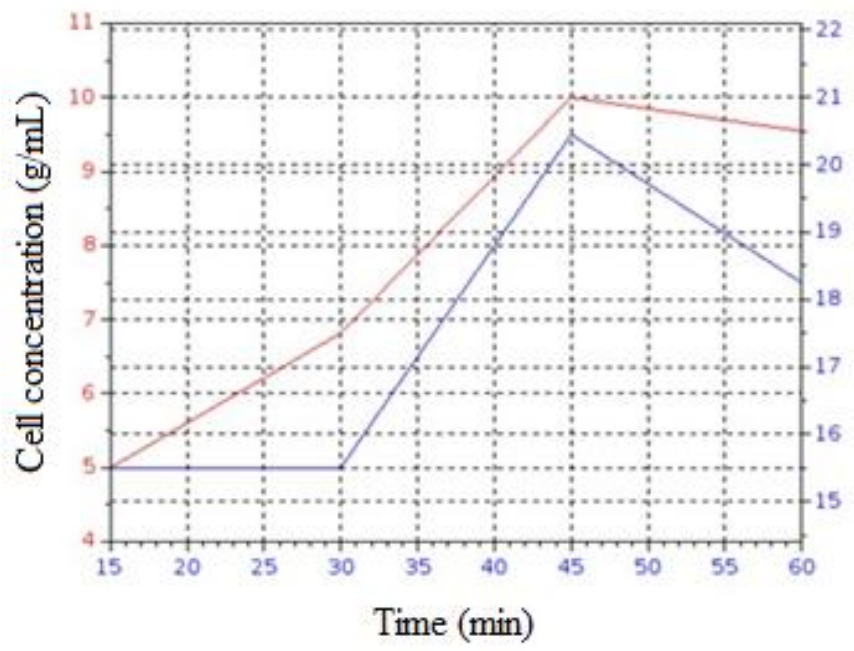

Figure 4: Experiment 1 - Values measured in red photos and analytical values in blue. 


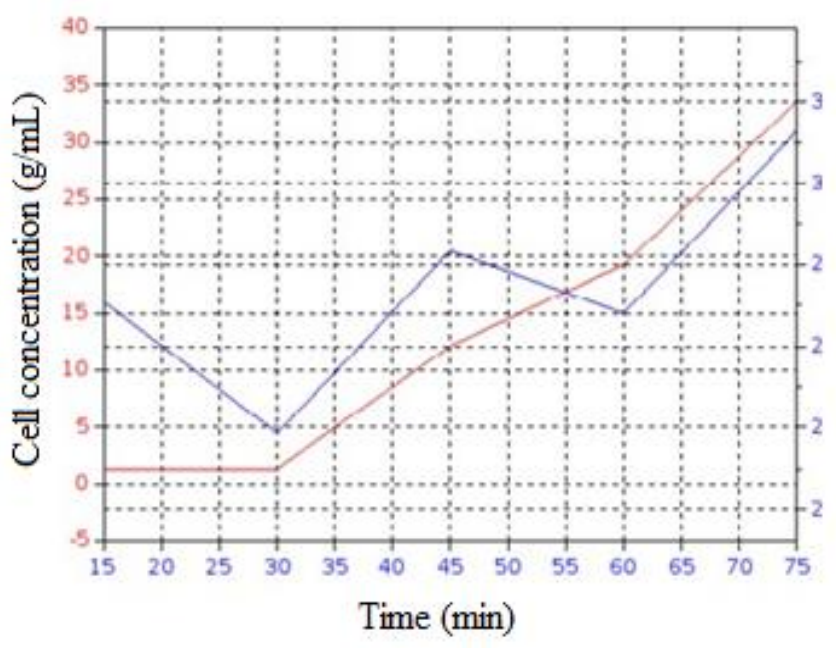

Figure 5: Experiment 2 - Values measured in red photos and analytical values in blue.

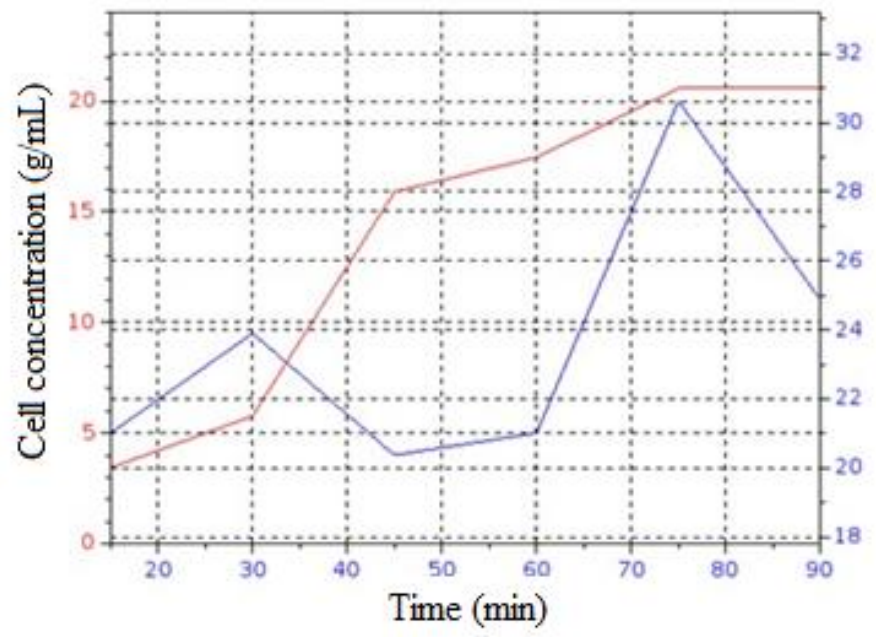

Figure 6: Experiment 3 - Values measured in red photos and analytical values in blue.

\section{Conclusion and suggestions for futures works}

From the figures 4, 5 and 6 it can be concluded that the method is promising for On-line analysis of concentrations of microorganisms in a fermentation process. If we continue to search for better correlations between images and analytical concentration data, we will find even better correlations than those presented in this paper. This is what we will do in the future and it is also a suggestion of future work.

In this work, only the time series of growth of the microorganism was used, in other works, one can use the analytical data and the images for the concentration of products and substrates. It is probable that the methodology of image processing is close to that developed in this work. 


\section{References}

[1] Souza J. S.; Identificação de Viabilidade de leveduras com corante vital utilizando histogramas de palavras visuais em imagens coloridas. Dissertação de Mestrado, Universidade Federal do Mato Grosso do Sul, 2015.

[2]Mongelo, A. I.; Validação de Método Baseado em Visão Computacional para Automação da Contagem de Viabilidade de Leveduras em Indústrias Alcooleiras. Dissertação de mestrado, Universidade Católica Dom Bosco, 2012.

[3] Gonzales, R. and woods, R. Digital Image Processing, Addison Wesley, 1993.

[4] Gabor, D. Theory of Communication. J.IEE (London), Vol. 93, pp. 429-457, 1946.

[5] Daubechies, I. Ten Lectures on Wavelets, Vol. 61, CBMS-NSF Regional Conference, 1992.

[6] Yamashita, Y. On-line measurement os cell size distribution and concentration of yeast by image processing. J. Chem. Eng. Of japan, Vol. 6, pp. 615-619, 1993.

[7]Luder, C. et al. In situ microscopy and MIR-spectroscopy as non-invasive optical sensors for cell cultivation process monitoring. Pharm. Bioprocess, Vol. 2, pp.157$166,2014$.

[8] Gutierrez, L.E. Produção de álcoois superiores por linhagens de Saccharomyces durante a fermentação alcoólica. Scientia Agricola, Vol. 50, pp. 464-472, 1993.

[9] Borzani, W. Batch ethanol fermentation: the correlation between the fermentation efficiency and the biomass initial concentration depends on what is considered as produced ethanol. Brazilian Journal of Microbiology, Vol. 37, pp. 87-89, 2006.

[10] Meyer, Y. Wavelets: Algorithms and Applications. Society for Industrial and Applied Mathematics, Philadelphia, first edition, 1993.

[11]Pugari, J. D. et al. Automatic Fungal Disease Detection based on Wavelet Feature Extraction and PCA Analysis in Commercial Crops. I.J. Image, Graphics and Signal Processing, Vol. 1, pp. 24-31, 2014.

\section{Acknowledgments}

The authors would like to thank Coordenação de Aperfeiçoamento de Pessoal de Nível Superior (CAPES) for the financial support. 